\title{
Technikgeschichte des Kalten Krieges
}

\author{
Eine einführende Skizze
}

VON KARIN ZACHMANN

Der Zusammenhang von Technik und Krieg ist ein klassisches Thema der Technikgeschichte. Aber die Frage, in welchem Maße die Technik unserer Gegenwart durch den über vier Jahrzehnte andauernden Kalten Krieg geprägt wurde, der vor zwei Jahrzehnten zu Ende ging, beschäftigt die Forschung, zumindest im deutschsprachigen Raum, erst seit Kurzem. Es gibt freilich eine umfangreiche Literatur zur Geschichte der Technik in jener Epoche, die der bedeutende britische Historiker Eric Hobsbawm ausdrücklich als eine Zeit des Krieges charakterisierte. Hobsbawm zog zur Begründung seiner Ansicht, dass jenes Zeitalter durchaus den Charakter eines Dritten Weltkrieges gehabt habe, den Philosophen Thomas Hobbes heran, der in seinem Leviathan 1651 formulierte: „Krieg besteht nicht nur aus Schlachten oder Kampfhandlungen, sondern auch aus einer Zeitspanne, in der der Wille, sich zu bekriegen, ausreichend vorhanden sei." ${ }^{\text {"1 }}$ Eine solche Zeitspanne, so Hobsbawm, sei die Zeit zwischen dem Abwurf der Atombomben auf Hiroshima und Nagasaki und dem Zusammenbruch der Sowjetunion gewesen, eine Zeit, in der ganze Generationen in Furcht vor der atomaren Eskalation des Konflikts der beiden aus dem Zweiten Weltkrieg hervorgegangenen Supermächte lebten. Andere Autoren sprechen von einer Zeit des „Nicht-Friedens“. In diesem Sinne verwandte George Orwell im Oktober 1945 den Begriff des Kalten Krieges zum ersten Mal, als er in dem Artikel You and the Atomic Bomb festhielt, dass die Atombombe wohl Kriege im großen Maßstab beendet habe, aber um den Preis der unendlichen Verlängerung eines Friedens, der kein Frieden sei. ${ }^{2}$ Wenig später brachte der französische Philosoph und Soziologe Raymond Aron den Charakter des Kalten Krieges auf die knappe Formel: „paix impossible, guerre improbable“. ${ }^{3}$ Frieden war unmöglich, wegen der Unversöhnlichkeit der Weltanschauungen der beiden Supermächte und ihrer Verbündeten. Eine umfassende kriegerische Auseinandersetzung aber war in Anbetracht der Gefahr einer nuklearen Vernichtung der Welt unwahrscheinlich. Gleichwohl rechneten die gesellschaftlichen Eliten ebenso wie alle Menschen durchaus mit dem Risiko eines Nuklearkrieges, der in beiden Militärblöcken Mitte der

1 Hobbes zitiert in Eric Hobsbawm, Das Zeitalter der Extreme. Weltgeschichte des 20. Jahrhunderts, München 1999, S. 286.

2 George Orwell, You and the Atomic Bomb, in: Tribune, GB, London, 19.10.1945.

3 Raymond Aron, Le Grand Schisme, Paris 1948, S. 13, Zitat aus John Lamberton Harper, The Cold War, New York 2011, S. 1. 
1950er Jahre zur strategischen Maxime erhoben worden war. Das aber hatte nicht nur weitreichende Auswirkungen auf die Gestaltung internationaler Politik und das komplexe Gefüge der internationalen Beziehungen, sondern wirkte sich auch maßgeblich auf die Technikentwicklung aus.

Während sich die Zeitgeschichte seit Jahrzehnten intensiv mit der historischen Analyse des Kalten Krieges beschäftigt, war das in der Geschichte der Technik lange Zeit kaum der Fall. ${ }^{4}$ Wie aber sollte die Technikgeschichte des Kalten Krieges erforscht und geschrieben werden? Wenn wir festhalten, dass die Spezifik internationaler Machtverhältnisse das Wesen des Kalten Krieges ausmacht, und diese Spezifik in der sowohl im globalen als auch im nationalen Rahmen aufgeführten Bipolarität der ideologischen Auseinandersetzung und atomaren Bedrohung bestand, dann muss folgende Frage im Zentrum stehen: Wie hat einerseits die spezifische Konstellation der internationalen Beziehungen die Entwicklung der Technik im nationalen und transnationalen Kontext geprägt, und wie wurde andererseits der Verlauf der Systemauseinandersetzung durch die Dynamik von Technisierungsprozessen beeinflusst? Es geht also um das Verhältnis von Technik und Politik und damit auch um die Frage, inwieweit sich die Entscheidungsprozesse und Rationalitäten beider Bereiche vermischen und politische Probleme auf die Technik verlagert oder technische Probleme politisch entschieden wurden. Im Zentrum steht die KoKonstruktion von Technik und Kaltem Krieg. Daraus ergibt sich eine enge Verknüpfung von Technik- und Zeitgeschichte.

Ein signifikantes Beispiel für diese Vermischung und Überlagerung von Technik und Politik ist die Atombombe, die als technisches Artefakt durch ihre bloße Existenz die Politik veränderte, weil sie zu anderen Formen der internationalen Auseinandersetzung zwang. Ein anderes Beispiel für diese Überlagerungsprozesse ist der Militärisch-Industrielle Komplex (MIK), vor dem einer seiner prominentesten Konstrukteure, der amerikanische General und Präsident Dwight Eisenhower im Jahre 1961 in seiner Abschiedsrede als Präsident ganz ausdrücklich warnte, als einer Gefahr für die Demokratie und die Freiheit der Forschung. ${ }^{5}$ Hobsbawm hat das Phänomen des MIK auf die eingängige Formel gebracht, es seien die ständig anwachsenden Kollektive von Menschen und Ressourcen, die durch und von der Vorbereitung eines Krieges lebten. ${ }^{6}$ Der MIK umfasste zum einen Handlungskollektive aus

4 Als Einstieg in die umfangreiche zeithistorische Literatur vgl. z.B. Harper (wie Anm. 3); Melvyn Leffler u. Odd Arne Westad (Hg.), The Cambridge History of the Cold War, 3 Bde., Cambridge 2010; Detlef Junker (Hg.), Die USA und Deutschland im Zeitalter des Kalten Krieges 1945-1990. Ein Handbuch, 2 Bde., Stuttgart 2001; Bernd Greiner, Tim B. Müller u. Claudia Weber (Hg.), Macht und Geist im Kalten Krieg, Hamburg 2011; Bernd Stöver, Der Kalte Krieg, München 2003; Jost Dülffer, Cold War History in Germany, in: Cold War History 8, 2008, S. 135-156.

5 Eisenhower's Farewell Address to the Nation, January 17, 1961. Internet: http://mcadams. posc.mu.edu/ike.htm (Stand: 28.6.2013).

6 Hobsbawm (wie Anm. 1), S. 285. 
menschlichen und nichtmenschlichen Akteuren mit ihren jeweils spezifischen Handlungspotenzialen. So bestimmen Ressourcen (z.B. Uranvorräte) oder Produkte (z.B. Atombomben) die Handlungsstrategien der kalten Krieger ebenso wie militärische oder ideologische Vorstellungen, die mit Hilfe der Technik über Computersimulationen generiert wurden. Zum anderen vereinten diese Handlungskollektive Mitglieder aus ganz verschiedenen gesellschaftlichen Bereichen, die unterschiedlichen Imperativen folgten. So wurde dem Militär Verantwortung für Sicherheit, der Politik für das Wohl des Staates, der Industrie für wirtschaftliches Wachstum und der Wissenschaft und Technik für Erkenntnis und Effizienz zugewiesen. Dass Mitglieder dieser Bereiche an gemeinsamen Projekten arbeiteten, machte viele Aushandlungs- und Übersetzungsprozesse notwendig, und es gilt zu untersuchen, welche der bereichsspezifischen Handlungsrationalitäten entscheidungsrelevant oder ausgeschaltet wurden.

Daraus ergibt sich die Frage, in welchem Maße der MIK die nationalen Innovationssysteme seit dem Zweiten Weltkrieg transformiert. Inwieweit hat der MIK neue Vorstellungen über den Ablauf von Innovationsprozessen und Veränderungen in der Art und Weise der Wissensproduktion hervorgebracht, die als neues Modell der Wissensproduktion „Mode 2“, als Fließbandmodell der Innovation, ${ }^{7}$ als hoher Staatsanteil in der Finanzierung von Forschung und Entwicklung, ${ }^{8}$ als Veränderung der Arbeitsteilung zwischen Wissenschaft und Industrie, als Zunahme der nicht mehr frei kommunizierten Wissensbestände und als enormes Größenwachstum der Universitäten und ihrer Ausbildungsaufgaben sichtbar wurden? ${ }^{9}$ Während zum MIK in den USA bereits viele Untersuchungen vorliegen, gibt es über den MIK der anderen Supermacht bislang vor allem Studien zu partiellen Entwicklungen des Komplexes, aber noch keine systematische Untersuchung des Gesamtphänomens. ${ }^{10}$ Und auch

7 Glen Asner, The Linear Model, the US Department of Defense, and Golden Age of Industrial Research, in: Karl Grandin, Nina Wormbs u. Sven Widmalm (Hg.), The Science-Industry Nexus. History, Policy, Implications. Nobel Symposium 123, Sagamore Beach 2004, S. 3-30; Benoit Godin, The Linear Model of Innovation. The Historical Construction of an Analytical Framework, in: Project on the History and Sociology of S\&T Statistics. Working Paper No. 30, Montreal 2005.

8 Robert Bud u. Philip Gummet (Hg.), Cold War, Hot Science: Applied Research in Britain's Defence Laboratories, 1945-1990, London 1999.

9 Stuart W. Leslie, The Cold War and American Science: The Military-Industrial-Academic Complex at MIT and Stanford. New York 1993; Noam Chomsky, Laura Nadler, Immanuel Wallerstein, Richard C. Lewontin u. Richard Ohmann (Hg.), The Cold War and the University: Toward an Intellectual History of the Postwar Years, New York 1997; Karin Zachmann, Mobilisierung der Frauen. Technik, Geschlecht und Kalter Krieg in der DDR, Frankfurt 2004.

10 Vgl. z.B. David Holloway, Stalin and the Bomb. The Soviet Union and Atomic Energy, 1939-1956, New Haven 1994; Paul R. Josephson, Red Atom: Russia's Nuclear Power Program from Stalin to Today, New York 1999; Alexei Kojevnikov, Die Mobilmachung der sowjetischen Wissenschaft, in: Greiner et al. (wie Anm. 4), S. 87-107; Slava Gerovitch, From Newspeak to Cyberspeak: A History of Soviet Cybernetics, Cambridge 2002; Asif A. Siddiqi, Challenge to Apollo: The Soviet Union and the Space Race, 1945-1974, Washington DC 2000. 
über die Kooperation von Industrie, Wissenschaft und Militär im geteilten Deutschland ist noch wenig bekannt. ${ }^{11}$

Einen weiteren Schwerpunkt der Technikentwicklung des Kalten Krieges bildet die Erschließung neuer Räume zur Aufführung der Systemauseinandersetzung. Hier ist zum einen der nur mit erheblichem Aufwand an Wissen und Technik machbare Vorstoß auf die Ozeane, die Polarregionen, die Atmosphäre und den Weltraum in den Blick zu nehmen. ${ }^{12}$ Im Wettstreit um die Besetzung dieser Räume ging es um die Erschließung symbolischer und materieller Ressourcen, um damit wissenschaftlich-technische Überlegenheit zu demonstrieren, Aufklärungstätigkeit abzusichern, Freiraum für die Wahl militärstrategischer Optionen zu erhalten und den Frontenverlauf im Kalten Krieg zu definieren. Wie bei dieser Schaffung neuer, technogener Räume einerseits militärische, machtpolitische und zivile Anliegen zusammenwirkten und sich zum anderen transnationale Netzwerke bilden, die möglicherweise auch die Frontstellungen im Kalten Krieg unterliefen, gilt es weiter zu untersuchen. Die Beiträge von Dania Achermann und Christian Kehrt in diesem Themenheft widmen sich diesen Fragen.

Doch nicht nur neu erschlossene, sondern auch bereits genutzte Räume avancierten zu Bühnen für die Inszenierung des Systemkonflikts. So wurden die Küchen, Bade- und Wohnzimmer, aber auch Supermärkte, Kaufhäuser, Kinos und Feriensiedlungen zur Arena des Ost-West-Vergleichs. Als sich der sowjetische Partei- und Staatschef Nikita Chruschtschow und der amerikanische Vizepräsident Richard Nixon im Juli 1959 in Moskau vor Tiegeln, Töpfen und Waschmaschine in einer von General Electric ausgestatteten Küche darüber stritten, ob Haushaltstechnik die Frauen befreie, waren die Weichen zur technischen Hochrüstung der individuellen Haushalte bereits gestellt. ${ }^{13}$ Seit 1949 tourten amerikanische Traumhaus- und Supermarktausstellungen durch Europa, die den fordistischen Gesellschaftsvertrag inszenierten. ${ }^{14}$ In welchem Ausmaß das amerikanische Modell auf beiden Seiten des Eisernen Vorhanges adaptiert wurde und wie hier die Infrastrukturen und die Hardware des energieintensiven, über ein ,immer mehr" an privaten Konsumgütern vermittelten Lebensstils entstanden, ist ein zentrales Thema der Technikgeschichte.

11 Aus Helmuth Trischler u. Rüdiger vom Bruch, Forschung für den Markt: Geschichte der Fraunhofer-Gesellschaft, München 1999, erfahren wir, dass die Fraunhofer-Gesellschaft bis zum Ende der 1960er Jahre zu ca. 50\% durch das Bundesverteidigungsministerium finanziert wurde.

12 Jacob Hamblin, Oceanographers and the Cold War: Disciples of Marine Science. Seattle 2005; Roger D. Launius, John M. Logsdon u. Robert W. Smith (Hg.), Reconsidering Sputnik. Forty Years since the Soviet Satellite, Amsterdam 2000; Siddiqi (wie Anm. 10). Auf weitere Literatur dazu wird in den Beiträgen von Achermann und Kehrt in diesem Heft verwiesen.

13 Ruth Oldenziel u. Karin Zachmann (Hg.), Cold War Kitchen. Americanization, Technology, and European Users, Cambridge 2009.

14 Greg Castillo, Cold War on the Home Front. The Soft Power of Midcentury Design, Minneapolis 2010 . 
Schließlich bildeten die Entwicklungsländer, die sich seit dem Ende der Kolonialherrschaft zu unabhängigen Staaten formierten, einen wichtigen Aufführungsraum des Kalten Krieges. Mit technischen Großprojekten wie Staudämmen, Kraftwerken, Fabriken und Infrastrukturen, der Errichtung Technischer Hochschulen, dem Transfer von Fachkräften und anderen Formen wissenschaftlich-technischer Hilfeleistungen, aber auch mit Technologieprojekten, für die die weniger entwickelten Länder als Versuchslabore funktionierten, konkurrierten die USA und die UdSSR darum, dort einerseits den Einfluss ehemaliger europäischer Kolonialmächte zurückzudrängen und zum anderen ihr Gesellschaftsmodell zu etablieren. Über die konkreten Konzepte, Organisationsformen, Abläufe, Aneignungsformen und Folgen dieser im Zeitalter der großen Auseinandersetzung zwischen der sogenannten Ersten und Zweiten Welt erfolgten Transfers von Wissen und Technik in die Räume der sogenannten Dritten Welt wissen wir aus technikhistorischer Perspektive noch viel zu wenig. ${ }^{15}$ Hier geht es auch darum, wie über Technik die Hierarchien in dieser Weltordnung, die sich in den Bezeichnungen klar manifestierten, hergestellt und perpetuiert oder aber auch punktuell ausgehebelt wurden. Von Interesse sind dabei auch die Arbeitsweise und Projekte der Vereinten Nationen mit ihren Spezialorganisationen, die großen Einfluss auf die globale Zirkulation von Wissen, Technik und Experten erlangten und dabei die Machtkonstellationen des Kalten Krieges mit bestimmten. ${ }^{16}$

Das bisher am besten bearbeitete Feld zur Technik im Kalten Krieg sind Untersuchungen zu speziellen Schlüsseltechnologien, die den Verlauf der Systemauseinandersetzung maßgeblich bestimmten. Dazu gehören die Kerntechnik, die Computertechnik und die Automatisierungstechnik. Die Kerntechnik stand im Zentrum des Wettrüstens. Parallel dazu forcierte die Eisenhower-Administration mit der Atoms-for-Peace-Initiative ihre Anwen-

15 Gabrielle Hecht (Hg.), Entangled Geographies: Empire and Technopolitics in the Global Cold War. Cambridge, MA 2011; Michael Adas, Dominance by Design, Cambridge 2006; Nick Cullather, The Hungry World: America's Cold War Battle Against Poverty in Asia, Cambridge, MA 2010.

16 Jessica Wang, The United States, the United Nations, and the Other Post-Cold War World Order: Internationalism and Unilateralism in the American Century, in: Ellen W. Schrecker (Hg.), Cold War Triumphalism: The Politics of American History After the Fall of Communism, New York 2004, S. 201-234; Darwin Hamblin, Let There Be Light ... And Bread: The United Nations, the Developing World, and Atomic Energy's Green Revolution, in: History and Technology 25, 2009, S. 22-48; Karin Zachmann, Risky Rays for an Improved Food Supply? National and Transnational Food Irradiation Research as a Cold War Recipe, Preprint Deutsches Museum Nr. 7, München 2013, Internet: http:/www.deutsches-museum.de/verlag/ aus-der-forschung/preprint/ [Stand: 30.4.2013]. Auch die Zeitgeschichte hat die Tätigkeit internationaler Organisationen als einen höchst relevanten Untersuchungsgegenstand für sich entdeckt. Iris Schröder, Die Wiederkehr des Internationalen. Eine einführende Skizze, in: Zeithistorische Forschungen/Studies in Contemporary History, Online-Ausgabe 8, 2011, H. 3, Internet: http://www.zeithistorische-forschungen.de/16126041-Editorial-3-2011 [Stand: 30.4.2013]. 
dung zu friedlichen Zwecken in der Energiegewinnung, Industrie, Landwirtschaft und Medizin, um damit eine internationale Kontrolle über das spaltbare Material und die Entwicklung der Kerntechnik zu errichten. ${ }^{17}$ Parallel dazu und unter Rückgriff auf Wissen aus dem US-Bombenprojekt forcierte die Sowjetunion den Ausbau der Kerntechnik in ihrem Einflussbereich. ${ }^{18}$ Viele Studien untersuchen diese Prozesse. ${ }^{19}$ Allerdings sind auch hier noch viele Fragen offen. So wissen wir noch wenig über die Vielzahl transnationaler Institutionen, die auf allen Gebieten der Kerntechnik wirksam waren und die Verteilung von Wissen und Macht in diesem für die Systemauseinandersetzung so sensiblen Gebiet beeinflussten.

Die Computertechnik und das Internet erwiesen sich aus mehreren Gründen als Schlüsseltechnologie des Kalten Krieges. Ihre Entwicklung bildete zum einen eine Grundvoraussetzung für den Rüstungswettlauf der Supermächte. Atombomben, Raketen und automatisierte Flug- und Raketenabwehrsysteme hätten ohne Computer nicht gebaut werden können. Zum anderen ermöglichten die rasant steigenden Rechenkapazitäten die mathematische Modellierung und Simulation von gesellschaftlichen Prozessen, so dass Computer und ihre Programmierer zu Akteuren einer technischen Konstruktion der sozialen Welt wurden. ${ }^{20}$ Schließlich avancierte die Computertechnik zu einem prägenden

17 John Krige, Atoms for Peace, Scientific Internationalism, and Scientific Intelligence, in: Osiris 21, 2006, S. 161-181; ders., The Peaceful Atom as Political Weapon: Euratom and American Foreign Policy in the Late 1950s, in: Historical Studies in the Natural Sciences 38, 2008, S. 5-44; Richard G. Hewlett u. Jack M. Holl, Atoms for Peace and War, 1953-1961: Eisenhower and the Atomic Energy Commission, Berkeley 1989.

18 Holloway (wie Anm. 10); Josephson (wie Anm. 10); Paul R. Josephson, Atomic Powered Communism: Nuclear Culture in the Postwar USSR, in: Slavic Review 55, 1996, S. 297-324; Alexei Kojevnikov, The Making of the Soviet Bomb and the Shaping of Cold War Science, in: Cathryn Carson u. David A. Hollinger (Hg.), Reappraising Oppenheimer: Centennial Studies and Reflections, Berkeley 2005, S. 129-145.

19 Für Deutschland z.B. Cathryn Carson, Nuclear Energy Development in Postwar West Germany: Struggles over Cooperation in the Federal Republic 's First Reactor Station, in: History and Technology 18, 2002, S. 233-270; Michael Eckert, Die Anfänge der Atompolitik in der Bundesrepublik Deutschland, in: Vierteljahreshefte für Zeitgeschichte 37, 1989, S. 115-143; Joachim Radkau, Aufstieg und Krise der deutschen Atomwirtschaft, Reinbek 1983; Johannes Abele u. Peter Liewers (Hg.), Zur Geschichte der Kernenergie in der DDR, Frankfurt a.M. 2000, S. 29-89; Johannes Abele, Kernkraft in der DDR. Zwischen Nationaler Industriepolitik und Sozialistischer Zusammenarbeit 1963-1990 (hg. vom Hannah-Ahrendt-Institut für Totalitarismusforschung e.V. an der TU Dresden, Berichte und Studien Nr. 26), Dresden 2000; Mike Reichert, Kernenergiewirtschaft in der DDR. Entwicklungsbedingungen, konzeptioneller Anspruch und Realisierungsgrad 1955-1990 (Studien zur Wirtschafts- und Sozialgeschichte, Bd. 17), St. Katharinen 1999.

20 Paul N. Edwards, The Closed World. Computers and the Politics of Discourse in Cold War America, Cambridge, MA 1996; Slava Gerovitch, ,Mathematical Machines' of the Cold War: Soviet Computing, American Cybernetics and Ideological Disputes in the Early 1950s, in: Social Studies of Science 31, 2001, S. 253-287; Gerovitch (wie Anm. 10); Hans Dieter Hellige, Militärische Einflüsse auf Leitbilder, Lösungsmuster und Entwicklungsrichtlinien der Computerkommunikation, in: Technikgeschichte 59, 1992, S. 371-401; Raúl Rojas u. 
Element des Konsumzeitalters. Seit den 1980er Jahren eroberten die Computer den privaten Raum. Sie wurden hier als eine Befreiungstechnologie wahrgenommen, die neue Zugänge zu Informationen und Dienstleistungen eröffnet. ${ }^{21}$ Das wiederum verschärfte den Ost-West-Gegensatz, weil die Demokratisierung der Computertechnik das staatliche Informationsmonopol des sozialistischen Blocks zu Fall brachte. Viele dieser Entwicklungen sind bereits Gegenstand der historischen Forschung, andere harren noch einer Untersuchung. So wissen wir noch zu wenig darüber, wie sich die Prozesse der Computerisierung in den einzelnen gesellschaftlichen Teilsystemen konkret durchsetzten und auswirkten und dadurch den Ausgang des Kalten Krieges beeinflussten.

Die Automatisierung ist ein weiteres Feld technischer Entwicklung, das in den Auseinandersetzungen des Kalten Krieges eine zentrale Rolle spielte. Die Automatisierung des Schlachtfeldes war für die Militärstrategien der USA von großer Bedeutung, um den Konflikt mit der amerikanischen Verfassung zu vermeiden, in der nur eine Defensivarmee vorgesehen war. ${ }^{22}$ Die politischen Führer des sozialistischen Lagers wiesen der Produktionsautomatisierung eine zentrale Bedeutung für die Systemauseinandersetzung zu, ausgehend von Lenins Behauptung, dass der Kommunismus den Kapitalismus besiegen wird, wenn er die höhere Arbeitsproduktivität erreicht hat. Für amerikanische Wirtschaftsführer wiederum war die Produktionsautomatisierung ein Weg, um die Gewerkschaften zu entmachten. Dass der Präsident von General Electrics Charles E. Wilson, der zeitweilig auch Präsident Eisenhower beriet, 1946 verkündete, „The problems of the United States can be captiously summed up in two words, Russia abroad, labor at home", macht die großen Erwartungen des Managements an die Automation schlagartig deutlich. ${ }^{23}$ Auch im geteilten Deutschland stand die Automatisierung Mitte der 1950er Jahre im Zentrum öffentlicher Debatten. Sie wurde auf beiden Seiten als eine Schlüsseltechnologie der zweiten Industriellen Revolution verstanden und mit Erwartungen an eine Annäherung der Systeme

Ulf Hashagen (Hg.), The First Computers - History and Architectures, Cambridge, MA, London 2000; Georg Trogemann, Alexander Y. Nitussov u. Wolfgang Ernst (Hg.), Computing in Russia. The History of Computer Devices and Information Technology Revealed, Braunschweig 2001.

21 Michael Friedewald, Der Computer als Werkzeug und als Medium. Die geistigen und technischen Wurzeln des Personal Computers (Aachener Beiträge zur Wissenschafts- und Technikgeschichte des 20. Jahrhunderts, Bd. 3), Berlin 1999.

22 Ausführlich dazu Edwards (wie Anm. 20).

23 David Hounshell, Automation, Transfer Machinery, and Mass Production in the U.S. Automobile Industry in the Post-World War II Era, in: Enterprise \& Society: The International Journal of Business History 1, 2000, S.100-138; David F. Noble, Forces of Production: A Social History of Industrial Automation, New York 1984; ders., Social Choice in Machine Design: The Case of Automatically Controlled Machine Tools, in: Merritt Roe Smith (Hg.), Military Enterprise and Technological Change. Perspectives on the American Experience, Cambridge MA 1987, S. 18-50. 
verknüpft. ${ }^{24}$ Wie diese unterschiedlichen Erwartungen die tatsächlichen Automatisierungsanstrengungen beeinflussten und zu welchen Ergebnissen sie führten, ist ein lohnendes Thema für eine Geschichte der Technik im Kalten Krieg.

Mit den hier vorgestellten Schwerpunkten sind die Untersuchungsfelder zur Technik im Kalten Krieg keineswegs erschöpft. Aber es sollte deutlich geworden sein, dass die Fokussierung auf diese historische Epoche ganz entschieden $\mathrm{zu}$ einer besseren Verknüpfung regionaler, nationaler und transnationaler Betrachtungsweisen zwingt, eine Perspektivierung und Ergänzung eurozentristischer bzw. auf den transatlantischen Raum begrenzter Studien befördert und die Technikgeschichte wieder stärker mit der Zeitgeschichte verknüpft.

Im Themenheft erscheinen Beiträge, die auf der Bochumer VDI-Tagung zum Thema „Technik im Kalten Krieg“ im Februar 2012 vorgestellt und danach für die Publikation vertieft und erweitert wurden. Die Aufsätze tragen wichtige Erkenntnisse zur Technikentwicklung im Kalten Krieg bei. So kann Mario Daniels in seinem Beitrag über die Herausbildung einer neuen USTechnologie- und Wissenspolitik in der Frühzeit des Kalten Krieges zeigen, wie sich als Teil der Umsetzung der amerikanischen Containment-Strategie die Prämissen für den Technologietransfer grundlegend wandelten. Während im Umgang mit den geistigen Reparationen aus Deutschland zunächst das mit der liberalen Staatsverfassung konforme Konzept einer Public Domain die amerikanische Wissens- und Technologiepolitik bestimmte, generierte die Containment-Strategie ein zunehmendes Sicherheitsbewusstsein, das durch die Aufdeckung sowjetischer Spionagetätigkeit in Schlüsselprojekten des MIK zusätzliche Nahrung erhielt. Wissens- und Technologietransfer wurden nun als Sicherheitsrisiko wahrgenommen und der Kontrolle unterworfen, wobei Exportkontrollen zum bevorzugten Instrument der sicherheitspolitischen Regulierung von Wissensströmen wurden.

Ein zentraler Aspekt der Technikentwicklung im Kalten Krieg war die Erschließung neuer Räume für die Systemauseinandersetzung. Dania Achermann untersucht die Forschungen und Experimente zur Wetterbeeinflussung im Kalten Krieg in der Bundesrepublik. Sie arbeitet heraus, dass die Techniken zur Wetterbeeinflussung als Formen der physischen und ideellen Besetzung der Atmosphäre zu begreifen sind, die damit als ein Machtraum instrumentalisiert wird. Wer das Wetter beeinflussen kann, beherrscht die Atmosphäre, was mit symbolischen und vielfältigen praktischen Bedeutungen verknüpft ist. Die Verfügbarkeit über einen schwer zugänglichen Raum symbolisiert hohe technisch-wissenschaftliche Leistungspotenziale, die auf der praktischen Ebene durch Wetterbeeinflussung zur Durchführung militärischer Aktionen, den Schutz vor Hagel in der Landwirtschaft oder die Sicherung des zivilen

24 Friedrich Pollock, Automation. Materialien zur Beurteilung der ökonomischen und sozialen Folgen, Frankfurt a.M. 1956; Leo Brandt, Die Zweite Industrielle Revolution. Macht und Möglichkeiten von Technik und Wissenschaft, München 1957; Fritz Selbmann, Ein Zeitalter stellt sich vor, Berlin (Ost) 1957. 
Flugverkehrs durch Nebelauflösung relevant wurden. Achermann zeigt, dass in den USA den militärischen Motiven der Wetterforschung die größte Bedeutung zukam, während in der Bundesrepublik ein wissenschaftliches Interesse und Anwendungen für lokalpolitische Belange dominierten. Gleichwohl war auch die bundesdeutsche Forschung zur Wetterbeeinflussung von den Strategien des Kalten Krieges geprägt, weil die Wissenschaftler zur internationalen Forschungsfront vorstoßen wollten, die sehr stark durch das militärisch motivierte US-Wissenschaftssystem dominiert war. Selbstverständlich galt Wetterbeeinflussung auch auf der anderen Seite des Eisernen Vorhanges als Ausweis wissenschaftlich-technischer Superiorität. In einem 1956 in der DDR erschienenen Kinderbuch erhielt ein Wettermacher das Wort, der im Jahre 1999 für das Wetter während der Leipziger Frühjahrsmesse verantwortlich sein sollte:

„wir meistern eine gewaltige Aufgabe. Die Menschheit ist stolz auf uns [...]. Entschlossen verhindern wir jeden gefährlichen Unfug, den das Wetter anstellen will. Wir bringen ein wenig Menschenverstand in das himmlische Chaos, wir können sogar ein krankes Klima allmählich kerngesund päppeln [...]. Das Messefestwetter ist unser stolzes, erhabenes Ausstellungsstück, worüber jeder kniefreie Münchner begeistert sein sollte! “25

Insgesamt ist über die Wetterforschung auf der östlichen Seite des Eisernen Vorhanges nur wenig bekannt.

Auch im Beitrag von Christian Kehrt geht es um die Besetzung neuer Räume in für den Menschen unwirtlichen Umwelten. Er untersucht, wie Grönland zu einem Labor des Kalten Krieges wurde, in dem militärische und zivile Akteure verschiedener Staaten aus durchaus unterschiedlichen Motiven heraus, aber in fruchtbarer Kooperation, Wissen produzierten und Technik erprobten. Als Vermittler für das Zusammenwirken erwiesen sich die gemeinsam genutzten Infrastrukturen, die vor allem von den USA für den Ausbau Grönlands zu ihrem wichtigsten Nuklear- und Militärstützpunkt außerhalb ihrer nationalen Grenzen bereitgestellt wurden. Kehrt zeigt auf, dass der Erfolg französischer Polarexpeditionen, die in einer zivilen Forschungstradition standen und dabei an deutsche Expeditionen aus den 1930er Jahren anknüpften, auf der gemeinschaftlichen Nutzung dieser Infrastrukturen beruhte. Das war mit Datenaustausch und punktueller Zusammenarbeit verbunden, die das auf globale Kontrolle ausgerichtete Stützpunktsystem der USA bestärkten. Christian Kehrt sieht seine Befunde als eine Bestätigung für die Auffassung von John Krige, der die These vertritt, dass die amerikanische Hegemonie im Bereich der Wissenschaft von den (West-)Europäern nicht nur akzeptiert, sondern auch mit hergestellt wurde.

Anschrift der Verfasserin: Prof. Dr. Karin Zachmann, Fachgebiet für Geschichte der Technik, Technische Universität München, c/o Deutsches Museum, 80306 München, E-mail: karin.zachmann@mzwtg.mwn.de

25 Werner Bender, Messeabenteuer 1999, Berlin 1956, S. 44. 
